PAPER

\title{
A Multiobjective Optimization Dispatch Method of Wind-Thermal Power System
}

\author{
Xiaoxuan GUO $^{\dagger, \dagger \dagger a)}$, Renxi GONG ${ }^{\dagger}$, Haibo BAO ${ }^{\dagger \dagger \dagger}$, Nonmembers, and Zhenkun LU ${ }^{\dagger \dagger \dagger b)}$, Member
}

SUMMARY It is well known that the large-scale access of wind power to the power system will affect the economic and environmental objectives of power generation scheduling, and also bring new challenges to the traditional deterministic power generation scheduling because of the intermittency and randomness of wind power. In order to deal with these problems, a multiobjective optimization dispatch method of wind-thermal power system is proposed. The method can be described as follows: A multiobjective interval power generation scheduling model of wind-thermal power system is firstly established by describing the wind speed on wind farm as an interval variable, and the minimization of fuel cost and pollution gas emission cost of thermal power unit is chosen as the objective functions. And then, the optimistic and pessimistic Pareto frontiers of the multi-objective interval power generation scheduling are obtained by utilizing an improved normal boundary intersection method with a normal boundary intersection (NBI) combining with a bilevel optimization method to solve the model. Finally, the optimistic and pessimistic compromise solutions is determined by a distance evaluation method. The calculation results of the 16-unit 174-bus system show that by the proposed method, a uniform optimistic and pessimistic Pareto frontier can be obtained, the analysis of the impact of wind speed interval uncertainty on the economic and environmental indicators can be quantified. In addition, it has been verified that the Pareto front in the actual scenario is distributed between the optimistic and pessimistic Pareto front, and the influence of different wind power access levels on the optimistic and pessimistic Pareto fronts is analyzed.

key words: interval multi-objective optimization, improved normal boundary intersection method, bilevel optimization, optimistic Pareto optimal front, pessimistic Pareto optimal front

\section{Introduction}

In the context of the growing global energy crisis and environmental pollution, clean energy is of high demand of future power system. Wind power technology, with clean and renewable advantages, has gained rapid development over the last decade, and the scale of electricity generation capacity has greatly increased year by year. Wind power is conducive to the realization of the adjustment of primary energy structure of the power grid, and the improvement of

\footnotetext{
Manuscript received July 22, 2020.

Manuscript revised August 13, 2020.

Manuscript publicized September 18, 2020.

${ }^{\dagger}$ The authors are with School of Electrical Engineering, Guangxi University, Nanning 530004, Guangxi, China.

${ }^{\dagger}$ The author is with Electric Power Research Institute, Guangxi Power Grid Corporation, Nanning 530023, Guangxi, China.

${ }^{\dagger \dagger}$ The author is with Nanning Power Supply Bureau, Guangxi Power Grid Corporation, Nanning 530031, Guangxi, China.

${ }^{+1+\dagger}$ The author is with College of Electronic Information, Guangxi University for Nationalities, Nanning 560006, Guangxi,
} China.

a) E-mail: guo_xiaoxuan@163.com (Corresponding author)

b) E-mail: lzk06@sina.com

DOI: $10.1587 /$ transinf.2020EDP7146 the environmental benefits of the power grid. However, its large-scale grid-connection will also increase the difficulty of power generation scheduling due to its intermittency and randomness [1]-[4], and makes it difficult to adapt to the traditional deterministic dispatching system.

It has become a hot issue to reasonably consider the uncertainty of wind power generation and seek a more perfect power dispatch scheme. According to different descriptions of the uncertainty, the schemes of wind power dispatching optimization can be categorised into the following two aspects:

(1) The stochastic optimization method [5]-[12]. Generally speaking, the prediction error of wind speed or wind speed is expressed as a random distribution. Based on the random scene of wind speed, chance constraints can be considered in the model. In the studies of [5]-[8], the wind speed is described as a random variable that obeys the Weibull distribution, an economic dispatch model with respect to the probability of simultaneous wind energy and solar energy power generation has been established, and the scheduling scheme under different operating conditions has been examined. In [9]-[12], a model of chance constrained economic dispatch is built by taking the deviation between the actual output and predictive value of wind power as random variables, and the scheduling scheme with certain probability is obtained. Although the stochastic optimization method is widely used at present, and there is much literature about it, the random optimization method needs to know the exact random distribution of wind speed, and it is usually difficult to accurately fit the random distribution of wind speed due to the limitation of historical data. At the same time, the reasonable construction of wind power scene lacks theoretical proof, which is also one of the limiting factors of the application of this method.

(2) The interval or robust optimization. In [13]-[16], the wind speed is modeled as an interval value, with the focus on the influence of the uncertainty of wind power intervals on the optimal solution (optimistic solution) and the worst solution (pessimistic solution). Based on the Stackelberg game theory, the optimal scheduling problem of power generation with wind power under the interval extreme scenario is studied in Ref. [16]. In [17], [18], the general modeling method of wind power uncertainty set is proposed by robust optimization. In the interval or robust optimization method, the wind power is modeled as an uncertain set, the extension information of uncertainty value is only needed to input, and few statistics are needed. Therefore, modeling is relatively 
easy.

However, for the above two types of method, it is difficult to accurately fit the probability distribution of wind speed or the output of wind power, which greatly affects the accuracy of the stochastic economic dispatch model.

In addition, up to now, in the researches on the wind power optimal scheduling [19]-[22] has rarely been considered the impact of wind power interval uncertainty on multiple optimization objectives such as economical and environmental objectives of the scheduling scheme. Although the multi-objective interval optimization problem has been studied in [23], it is only solved by simple interval operation, and the result is not accurate.

Therefore, in order to be able to consider the uncertainty of wind power and multi-objective optimal scheduling objectives at the same time, an optimal dispatch method of wind-thermal power system is proposed. With the wind speed described as interval variable and the fuel cost of thermal power unit and emission cost of gaseous contaminants acted as objective functions, a multi-objective interval power generation dispatch model of wind-thermal power system is firstly established. Meanwhile, in order to more effectively solve the problem, an improved normal boundary intersection (NBI) method is proposed by appropriately combining the interval optimization technique and normal boundary intersection method.

To be more specific, firstly, the improved NBI is used to convert the multi-objective interval optimization problem into a set of single-objective interval optimization problems. Next, two deterministic mathematical optimal models are generated by using the two-level combinatorial optimization to reprogram each single-objective interval optimization. Furthermore, the deterministic optimization model is solved by introducing the interior point method, and two Pareto solutions, namely the optimistic and pessimistic solutions, are obtained respectively. Lastly, the optimistic and pessimistic Pareto frontiers of the multi-objective interval optimization are constructed according to the optimistic Pareto solution and the pessimistic Pareto solution of each single objective interval, and optimistic and pessimistic compromise solution of these two Pareto results is finally figured out through the distance evaluation method.

The calculation results of the 16-unit 174-bus real system show that the uniform optimistic and pessimistic Pareto optimal solution set can be obtained by the established model and proposed method, which can be used to quantitatively analyze the impact of wind power on the economic and environmental cost of power generation dispatching, thus providing the reference for the actual dispatching decision of power system.

\section{A Multi-Objective Interval Optimization Model for Wind-Thermal Power Generation Dispatching}

\subsection{Interval Model of Wind Farm}

The output power of wind farm is the sum of the output power of each wind turbine generator, and the output power of the wind turbine is stochastic because of the random fluctuation of the wind speed. Assume that the wind speed of each wind turbine unit is the same at the same time, the output active power of the wind farm can be expressed as:

$$
\begin{aligned}
& P_{W}=n_{W} P_{W g} \\
& P_{W g}= \begin{cases}0, & v \leq v_{\text {in }}, v>v_{\text {out }} \\
a+b v, & v_{\text {in }} \leq v \leq v_{r} \\
P_{r}, & v_{r} \leq v \leq v_{\text {out }}\end{cases}
\end{aligned}
$$

Where, $P_{W}$ is the active output of wind farm, $n_{W}$ is the number of wind turbines on wind farms, $P_{W g}$ stands for the active output of a single wind turbine, $v$ represents the actual wind speed of wind farm, $v_{i n}, v_{\text {out }}$, and $v_{r}$ are the cut-in wind speed, cut-out wind speed, and rated wind speed, respectively; $P_{r}$ is the rated output power of wind turbines; $P_{W g}$ is the actual output power of wind turbine; $a=\frac{P_{r} v_{i n}}{v_{i n}-v_{r}}$ and $b=\frac{P_{r}}{v_{r}-v_{\text {in }}}$ are constants.

Based on the boundary information of wind speed fluctuation obtained from a large number of statistical data, the wind speed interval variable can be defined as:

$$
v^{ \pm}=\left[v^{-}, v^{+}\right]
$$

Where, $v^{+}$and $v^{-}$are upper and lower borders of $v^{ \pm}$, the actual wind speed $v$ can be an arbitrary value in the interval $\left[v^{-}, v^{+}\right]$. Due to the uncertainty of the interval of wind speed, the output powers of wind turbine and wind farm are both interval variables according to Eqs. (1) and (2). Therefore, they can be expressed as $P_{W g}^{ \pm}, P_{W}^{ \pm}$. It should be noted that the input condition of the proposed model is the wind speed range which is known, and $P_{W g}^{ \pm}$and $P_{W}^{ \pm}$are the intermediate variables of the model.

\subsection{Model of Wind-Thermal Power Generation Dispatch- ing}

Based on the assumption that all the wind power output is from the electric power system, taking the economic cost and the minimum environmental cost as the objective function, a multi-objective interval generation scheduling model is set up as follows:

\section{(1) Objective functions}

The minimization of the economic cost of thermal power unit $f_{C}$, which refers to the objective function of the minimum coal consumption cost:

$$
\min f_{C}=\sum_{i=1}^{N_{T}}\left(a_{i} P_{T i}^{2}+b_{i} P_{T i}+c_{i}\right)
$$

Where, $P_{T i}$ is the active power output of the $i$ th thermal power unit; $a_{i}, b_{i}$ and $c_{i}$ are parameters of economic cost of the $i$ th thermal power turbine, and $N_{T}$ refers to the number of the $i$ th thermal power units in the power system. The minimization of the environmental cost of thermal power unit $f_{E}$, namely, the objective function of the emission of 
polluting gases of the power unit is:

$$
\min f_{E}=\sum_{i=1}^{N_{T}}\left(\alpha_{i} P_{T i}^{2}+\beta_{i} P_{T i}+\gamma_{i}+\delta_{i} e^{\lambda_{i} P_{T i}}\right)
$$

Where, $\alpha_{i}, \beta_{i}, \gamma_{i}, \delta_{i}$ and $\lambda_{i}$ are the coefficients of pollution discharge of the thermal power unit.

(2) Constrained conditions

The equilibrium constraint of system power to be considered is the constraint with the network loss ignored, that is:

$$
\sum_{i=1}^{N_{T}} P_{T i}+\sum_{i=1}^{N_{N}} P_{W i}^{ \pm}-P_{D}=0
$$

Where, $P_{D}$ is the total active load of power system. The uncertain output of wind power interval can be determined by Eqs. (1) to (3).

The output constraint of each thermal power unit is:

$$
\underline{P}_{T i} \leq P_{T i} \leq \bar{P}_{T i} ; \quad i=1, \mathrm{~L}, N_{T}
$$

Where, $\underline{P}_{T i}$ and $\bar{P}_{T i}$ are the lower and upper bounds of the active output of the $i$ th thermal power unit.

The positive spinning reserve capacity constraint is:

$$
\sum_{i=1}^{N_{T}}\left(\bar{P}_{T i}-P_{T i}\right) \geq U_{S R}
$$

Where, $U_{S R}$ is the standby requirement for the routine system operation. The output of one of the largest thermal power units is usually given for the current period, that is $U_{S R}=\max \left\{\bar{P}_{T i}\right\}$.

If the network constraints and security constraints of the system need to be considered, then the distributed transfer factor of the network can be used to transform the branch power constraint into the node injection power constraint. This is not the focus of this study, thus not being considered in the above model.

\section{Solution of Multi-Objective Interval Optimization}

In the multi-target interval generation scheduling model, the interval uncertainty of wind speed needs to be taken into account, so that the objective function and the generating capacity of each unit will be the interval quantities. Meanwhile, the environmental cost and economic cost are taken as the objective functions, and what is sought is the multiobjective optimal solution of network power balance equation and physical limitation of electrical equipment satisfied.

By utilizing the traditional multi-objective generation scheduling, a solution set satisfying Pareto can be obtained. That is, Pareto optimal frontier. It should be noted that each solution on the Pareto frontier is a definite quantity. Because of the interval uncertainty of wind speed, by the scheduling, two Pareto frontiers can be gained: one of the best Pareto frontiers, that is, optimistic Pareto frontier; and relatively, a worst Pareto frontier, namely, pessimistic Pareto frontier. According to the actual wind velocity fluctuation scenario, the actual Pareto frontier is necessarily distributed between the optimistic Pareto frontier and the pessimistic Pareto frontier.

The general model Eqs. (4)-(8) of the multi-objective interval power dispatching can be reframed as the following:

$$
\begin{array}{ll}
\min & \left(f_{1}\left(x, u^{ \pm}\right), f_{2}\left(x, u^{ \pm}\right)\right) \\
\text {s.t. } & h\left(x, u^{ \pm}\right)=0 \\
& g\left(x, u^{ \pm}\right) \leq 0
\end{array}
$$

Where, $f_{1}$ and $f_{2}$ are the objective functions of the multiobjective interval optimization; $h\left(x, u^{ \pm}\right)=0$ and $g\left(x, u^{ \pm}\right) \leq$ 0 are the equality and inequality constraint of the model, respectively; $x$ is the adjustable quantity of the multi-objective optimization, that is, the unknown solution; $u^{ \pm}$is the indefinite quantity, whose average is $u^{0}=\left(u^{-}+u^{+}\right) / 2$.

Obviously, Eq. (9) can not be directly solved. In this paper, we propose a method for solving it. By the method, the solution process can be outlined as follows: firstly, the improved NBI method is used to transform it into a single objective optimization problem, and then, the interval optimization method is utilized to determine the solution of each single objective optimization problem, thus determining the Pareto frontier of the problem; finally, the distance evaluation method is employed to optimize the optimistic Pareto compromise and pessimistic Pareto compromise solution.

\section{Improved NBI Method}

The NBI method can be used to construct a relatively uniform Pareto frontier by means of a space transformation and projection intercept [24], [25], which has good application effect in solving the multi-objective optimization problem of power system. The main steps of the NBI method [26][28].

The main improvements made are as follows: (1) The interval uncertainty of $u^{ \pm}$is used to obtain the mean $u^{0}$, the endpoints of the Utopia line are determined and the normalized space of the objective function is established. (2) The optimal intercept problem is not considered as the deterministic optimization problem, but the interval optimization problem, which can get two extremes of the optimistic and pessimistic Pareto frontier.

\subsection{Normalization of Objective Function}

By substituting the average $u^{0}$ of the interval uncertainty $u^{ \pm}$ into Eq. (9), a certain multi-objective optimization problem is obtained. With the $f_{1}$ and $f_{2}$ as the optimization objectives, the following two optimization problems are established:

$$
\begin{array}{ll}
\min & f_{1}\left(x, u^{0}\right) \\
\text { s.t. } & h\left(x, u^{0}\right)=0 \\
& g\left(x, u^{0}\right) \leq 0 \\
\min & f_{2}\left(x, u^{0}\right)
\end{array}
$$




$$
\begin{array}{ll}
\text { s.t. } & h\left(x, u^{0}\right)=0 \\
& g\left(x, u^{0}\right) \leq 0
\end{array}
$$

By solving Eqs. (10)-(11), the single objective optimal solutions $x^{1 *}$ and $x^{2 *}$ can be obtained. The line segment with $\left(f_{1}\left(x^{1 *}, u^{0}\right), f_{2}\left(x^{1 *}, u^{0}\right)\right)$ and $\left(f_{1}\left(x^{2 *}, u^{0}\right), f_{2}\left(x^{2 *}, u^{0}\right)\right)$ as the endpoints is called Utopia line.

In order to avoid the numerical problems caused by different dimensions of the objective functions, the space of the objective function can be normalized and transformed as follows:

$$
\left\{\begin{array}{l}
\bar{f}_{1}=\frac{f_{1}\left(x, u^{ \pm}\right)-f_{1}\left(x^{1 *}, u^{0}\right)}{f_{1}\left(x^{2 *}, u^{0}\right)-f_{1}\left(x^{1 *}, u^{0}\right)} \\
\bar{f}_{2}=\frac{f_{2}\left(x, u^{ \pm}\right)-f_{2}\left(x^{2 *}, u^{0}\right)}{f_{2}\left(x^{1 *}, u^{0}\right)-f_{2}\left(x^{2 *}, u^{0}\right)}
\end{array}\right.
$$

Where, $\bar{f}_{1}$ and $\bar{f}_{2}$ are normalized objective functions, $f_{1}\left(x^{1 *}, u^{0}\right)$ and $f_{2}\left(x^{2 *}, u^{0}\right)$ are the best points, and $f_{1}\left(x^{2 *}, u^{0}\right)$ and $f_{1}\left(x^{2 *}, u^{0}\right)$ are the worst points.

\subsection{Normal Vector Projection}

Figure 1 is a functional space that is normalized according to Eq. (12). It is assumed that the line segment between Utopia segments is divided into $m$ segments, and the coordinate $\left(a_{i}, b_{i}\right)$ of the endpoints $X_{i} ; i=0,1, \mathrm{~L}, m$ of each line segment can be defined as:

$$
\left(a_{i}, b_{i}\right)=\beta_{2}(0,1)+\beta_{1}(1,0)=\left(\beta_{1}, \beta_{2}\right)
$$

Where, $\beta_{1}$ and $\beta_{2}$ are the weights of the two endpoints, respectively. From Fig. 1, the further determination can be made:

$$
\left(a_{i}, b_{i}\right)=\beta_{2}(0,1)+\beta_{1}(1,0)=\left(\beta_{1}, \beta_{2}\right)
$$

\subsection{Solution of the Intercept Optimization Problem}

With the point $X_{i}$ of the Utopia line as the starting point, along the normal direction of the Utopia line, the point of

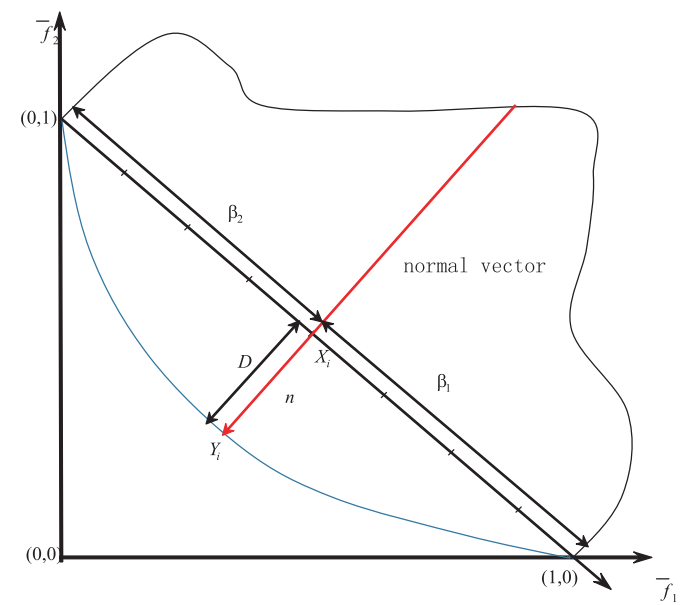

Fig. 1 Pareto frontier and utopia line of normalized objectives. intersection with the Pareto front is just the optimal solution $Y_{i} ; i=0,1, \cdots, m$ of the multi-objective optimization problem (10)-(11). The normal vector is $\vec{n}=-(1,1)$, from which the coordinates of $Y_{i}$ can be derived as $\left(c_{i}, d_{i}\right)$ :

$$
\left(c_{i}, d_{i}\right)=\left(a_{i}, b_{i}\right)-D_{i} \stackrel{r}{n}=\left(a_{i}-\frac{1}{\sqrt{2}} D_{i}, b_{i}-\frac{1}{\sqrt{2}} D_{i}\right)
$$

Where $D_{i}$ is the distance between point $X_{i}$ and point $Y_{i}$, which stands for the maximum distance from the Utopia line to the target spatial domain boundary. Since $c_{i}$ and $d_{i}$ are the normalized objective functions $\bar{f}_{1}$ and $\bar{f}_{2}$, from Eq. (12), Eq. (15) can be organized as:

$$
\left\{\begin{array}{l}
\frac{f_{1}\left(x, u^{ \pm}\right)-f_{1}\left(x^{1 *}, u^{0}\right)}{f_{1}\left(x^{2 *}, u^{0}\right)-f_{1}\left(x^{1 *}, u^{0}\right)}-\frac{i}{m}+\frac{1}{\sqrt{2}} D_{i}=0 \\
\frac{f_{2}\left(x, u^{ \pm}\right)-f_{2}\left(x^{2 *}, u^{0}\right)}{f_{1}\left(x^{1 *}, u^{0}\right)-f_{1}\left(x^{2 *}, u^{0}\right)}-1+\frac{i}{m}+\frac{1}{\sqrt{2}} D_{i}=0
\end{array}\right.
$$

According to Eq. (15), the optimal solution on the front edge of Pareto is determined by the distance $D_{i}$. Having obtained all the optimal solutions $Y_{i}$, the Pareto front of the multiobjective optimization problem can be described. Thus, the original multi-objective optimization problem can be transformed into the following $m+1$ single-objective optimization problems:

$$
\begin{array}{ll}
\min & -D_{i} \\
\text { s.t. } & \frac{f_{1}\left(x, u^{ \pm}\right)-f_{1}\left(x^{1 *}, u^{0}\right)}{f_{1}\left(x^{2 *}, u^{0}\right)-f_{1}\left(x^{1 *}, u^{0}\right)}-\frac{i}{m}+\frac{1}{\sqrt{2}} D_{i}=0 \\
& \frac{f_{2}\left(x, u^{ \pm}\right)-f_{2}\left(x^{2 *}, u^{0}\right)}{f_{2}\left(x^{1 *}, u^{0}\right)-f_{2}\left(x^{2 *}, u^{0}\right)}-1+\frac{i}{m}+\frac{1}{\sqrt{2}} D_{i}=0 \\
& h\left(x, u^{ \pm}\right)=0 \\
& g\left(x, u^{ \pm}\right) \leq 0
\end{array}
$$

Equation (17) contains interval uncertainty $u^{ \pm}$. The problem is a non-linear interval optimization problem. Obviously, due to the effects of the uncertain $u^{ \pm}$variables, the intercept values obtained by the interval variables should be $D_{i}=\left[D_{i}^{-}, D_{i}^{+}\right]$, which are the maximum intercept $D_{i}^{+}$and the minimum intercept $D_{i}^{-}$, and then the optimistic Pareto solution $Y_{i}^{+}$and pessimistic Pareto solution $Y_{i}^{-}$can be determined. Accordingly, the optimistic Pareto front and pessimistic Pareto front can be drawn out.

The interval programming problem of Eq. (17) can be transformed into two bilevel programming with maximum intercept problem and minimum intercept problem [29], specifically as follows:

(1) Maximum intercept problem

$$
\begin{array}{ll}
\min & -D_{i} \\
\text { s.t. } & u^{-} \leq u \leq u^{+} \\
& x \in \arg \min _{x}-D_{i} \\
& \text { s.t. } \\
& \frac{f_{1}(x, u)-f_{1}\left(x^{1 *}, u^{0}\right)}{f_{1}\left(x^{2 *}, u^{0}\right)-f_{1}\left(x^{1 *}, u^{0}\right)}-\frac{i}{m}+\frac{1}{\sqrt{2}} D_{i}=0 \\
& \frac{f_{2}(x, u)-f_{2}\left(x^{2 *}, u^{0}\right)}{f_{2}\left(x^{1 *}, u^{0}\right)-f_{2}\left(x^{2 *}, u^{0}\right)}-1+\frac{i}{m}+\frac{1}{\sqrt{2}} D_{i}=0
\end{array}
$$




$$
\begin{aligned}
& h(x, u)=0 \\
& g(x, u) \leq 0
\end{aligned}
$$

Equation (18) can be changed to the single-layer optimization problems as follows:

$$
\begin{array}{ll}
\min & -D_{i} \\
\text { s.t. } & u^{-} \leq u \leq u^{+} \\
& \frac{f_{1}(x, u)-f_{1}\left(x^{1 *}, u^{0}\right)}{f_{1}\left(x^{2 *}, u^{0}\right)-f_{1}\left(x^{1 *}, u^{0}\right)}-\frac{i}{m}+\frac{1}{\sqrt{2}} D_{i}=0 \\
& \frac{f_{2}(x, u)-f_{2}\left(x^{2 *}, u^{0}\right)}{f_{2}\left(x^{1 *}, u^{0}\right)-f_{2}\left(x^{2 *}, u^{0}\right)}-1+\frac{i}{m}+\frac{1}{\sqrt{2}} D_{i}=0 \\
& h(x, u)=0 \\
& g(x, u) \leq 0
\end{array}
$$

Equation (19) is a non-linear programming problem that can be solved directly using the original dual interior point algorithm [30].

(2) Minimum intercept problem

$$
\begin{aligned}
& \max -D_{i} \\
& \text { s.t. } \quad u^{-} \leq u \leq u^{+} \\
& x \in \arg \min _{x}-D_{i} \\
& \text { s.t. } \frac{f_{1}(x, u)-f_{1}\left(x^{1 *}, u^{0}\right)}{f_{1}\left(x^{2 *}, u^{0}\right)-f_{1}\left(x^{1 *}, u^{0}\right)}-\frac{i}{m}+\frac{1}{\sqrt{2}} D_{i}=0 \\
& \frac{f_{2}(x, u)-f_{2}\left(x^{2 *}, u^{0}\right)}{f_{2}\left(x^{1 *}, u^{0}\right)-f_{2}\left(x^{2 *}, u^{0}\right)}-1+\frac{i}{m}+\frac{1}{\sqrt{2}} D_{i}=0 \\
& h(x, u)=0 \\
& g(x, u) \leq 0
\end{aligned}
$$

Equation (20) is a bilevel non-linear programming problem, which can be transformed into a non-linear programming problem with complementary constraints by replacing its underlying problem with its dual feasible constraints. The detailed solution technology can be referred to [31].

\section{Choice of Compromise Solution}

Having obtained the optimistic Pareto frontier and pessimistic Pareto frontier by the improved NBI method and interval optimization method, the distance evaluation method [32] can be used to determine the optimistic Pareto and pessimistic Pareto compromise solution. In Fig. 1, for example, the distance indicator defined by Eq. (15) is as follows:

$$
\begin{aligned}
L_{i} & =\sqrt{c_{i}^{2}+d_{i}^{2}} \\
& =\sqrt{\left(a_{i}-\frac{1}{\sqrt{2}} D_{i}\right)^{2}+\left(b_{i}-\frac{1}{\sqrt{2}} D_{i}\right)^{2}} \\
& =\sqrt{\left(\frac{i}{m}-\frac{1}{\sqrt{2}} D_{i}\right)^{2}+\left(1-\frac{i}{m}-\frac{1}{\sqrt{2}} D_{i}\right)^{2}}
\end{aligned}
$$

the origin $(0,0)$, and the nearest solution from the origin is chosen as the compromise solution, which is the very solution satisfying $\min \left\{L_{i} ; i=0,1,2, \cdots, m\right\}$.

\section{Solution Procedure}

The specific steps of the multi-objective interval generation dispatching method can be described as follows:

(1) The multi-objective interval optimization model of the power dispatching of power system is established by introducing the interval distribution of the wind speed of the wind farm, as shown in Eqs. (4)-(8);

(2) The original multi-objective interval optimization problem is transformed into $m+1$ interval non-linear programming problems by using the improved NBI method, as shown in Eq. (17);

(3) The interval non-linear programming Eq. (17) is converted into a non-linear programming Eq. (19) and a bilevel non-linear programming Eq. (20). That is, the original multi-objective interval optimization is transformed to $m+1$ non-linear programming and $m+1$ bilevel non-linear programming frameworks;

(4) The original dual internal point algorithm and dual feasible constraint method are used to solve the problems of the non-linear programming and double-layer non-linear programming, thus obtaining $m+1$ maximum intercept $D_{i}^{+}$and minimum intercept $D_{i}^{-}$;

(5) According to the maximum intercept $D_{i}^{+}$and the minimum intercept $D_{i}^{-}, m+1$ optimistic Pareto solutions and $m+1$ pessimistic Pareto solutions are obtained, thus drawing out the optimistic Pareto frontier and pessimistic Pareto front;

(6) The distance evaluation method is used to determine the compromise solution of the optimistic Pareto and pessimistic Pareto.

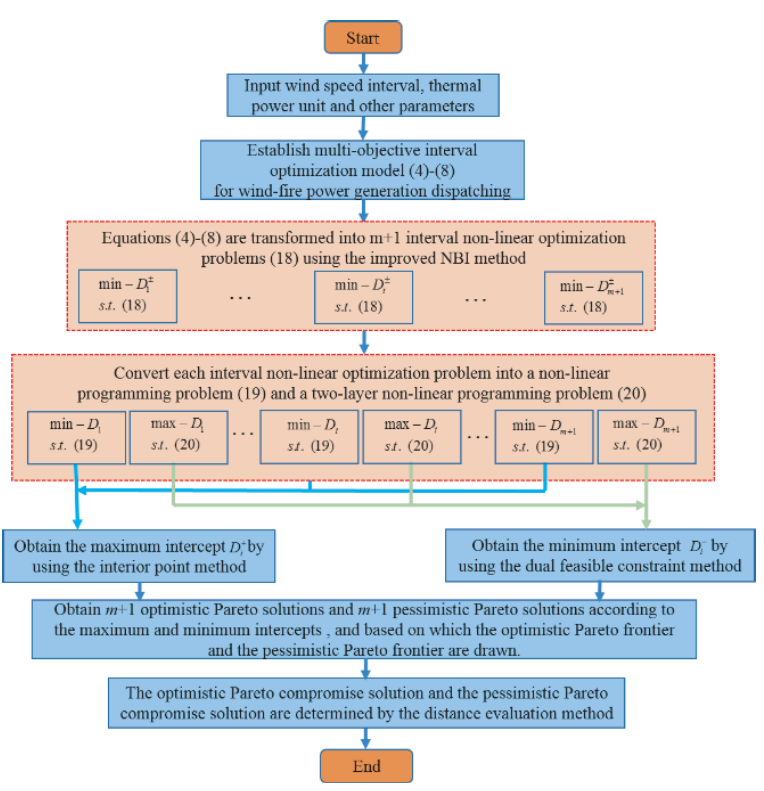

Fig. 2 Process of improved NBI method. 
The process of the improved NBI method is shown as in Fig. 2.

\section{Analysis of Calculation Results}

\subsection{System Overview}

In order to verify the effectiveness of the proposed method and built model, an experimental system is constructed. The configuration of the system is as follow: The CPU frequency of the computer platform is $3.2 \mathrm{GHz} \times 2$, Memory is $8 \mathrm{~GB}$. The selected test system is a 16-unit 174-bus system of a provincial power grid in China Southern Power Grid, and the total active load of the system is $4688 \mathrm{MW}$. The economic cost parameters of each thermal power generating unit and the cost parameters of the pollutant emission are shown in Table A. 1 (Schedule 1), and the wind farm parameters and air velocity prediction intervals are shown in Table A. 2 (Schedule 2).

\subsection{Analysis of Pareto Solution Set}

The wind speed of each wind field is set to the mean value of the forecast interval of Schedule 2. With the minimum economic cost and the minimum pollutant emission as the objective functions, the endpoints of the Utopia line determined by the improved NBI method are shown in Table 1.

The optimistic and pessimistic Pareto frontier of the multi-objective interval generation dispatching determined by the improved NBI method is shown as in Fig. 3, with the number of segments of the Utopia line set as $m=20$. Obviously, the optimistic and pessimistic Pareto frontiers obtained by the improved NBI method are evenly distributed in a two-dimensional coordinate system, and the dispatcher can select the optimal solution needed to set the scheduling scheme. The distance between the Pareto frontier and

Table 1 Extreme points of utopia line.

\begin{tabular}{lcc}
\hline \hline Extreme points & $f_{C} /(\$ / \mathrm{h})$ & $f_{E} /\left(10^{-4} \mathrm{t} / \mathrm{h}\right)$ \\
\hline minimum economic cost & 83.9131 & 746.5809 \\
minimum pollutant emission & 90.7392 & 469.2816 \\
\hline \hline
\end{tabular}

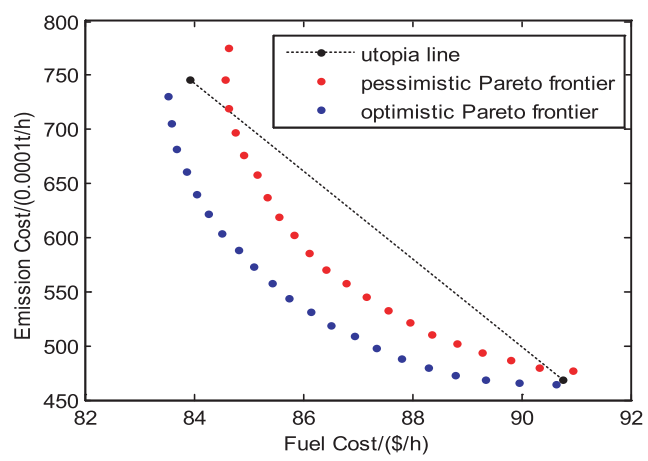

Fig. 3 Comparison of optimistic Pareto frontier and pessimistic Pareto frontier. the pessimistic Pareto frontier reflects the influence of the uncertainty of the wind speed interval on the result of the multiple-target interval generation scheduling.

The optimistic Pareto solution set and pessimistic Pareto solution set corresponding to Fig. 3 are shown in Table 2 and Table 3. The distance evaluation method is used to choose the compromise solution. Firstly, a sort of distance index Pareto solution is made, then, a selection of the minimum distance index solution as a compromise solution is performed, and then the optimistic Pareto compromise solution can be determined as $f_{\mathrm{OC}}=85.7541(\$ / \mathrm{h})$ and $f_{\mathrm{OE}}=544.0679\left(10^{-4} \mathrm{t} / \mathrm{h}\right)$ and the pessimistic Pareto compromise solution as $f_{\mathrm{PC}}=86.4197(\$ / \mathrm{h})$ and $f_{\mathrm{PE}}=571.1074$ $\left(10^{-4} \mathrm{t} / \mathrm{h}\right)$.

In order to verify the relationship between the Pareto frontier and the optimistic and pessimistic Pareto frontier under the actual wind speed scenario, three kinds of wind speed scenarios are chosen according to Schedule 1, whose wind speed and wind farm output power are shown in Table 4, and a deterministic multi-objective generation scheduling model is established. The Pareto frontiers obtained under the three scenarios are shown in Fig. 4. Clearly, all the Pareto frontiers of the three scenarios are distributed between the optimistic and pessimistic Pareto frontier. What should be stated here that the three scenarios in Fig. 4 are the arbitrarily chosen scenarios, and the wind farm output is arbitrarily selected in the interval, and the similar results can be obtained. It is possible to foresee that the actual Pareto

Table 2 Optimistic pareto frontier.

\begin{tabular}{lllll}
\hline \hline No. $i$ & $\mathrm{D}_{i}^{+}$ & $\mathrm{L}_{i}^{+}$ & $f_{\mathrm{OC}} /(\mathrm{S} / \mathrm{h})$ & $f_{\mathrm{O}} /\left(10^{-4} \mathrm{t} / \mathrm{h}\right)$ \\
\hline 0 & 0.0821 & 0.9437 & 83.5168 & 730.4827 \\
1 & 0.1359 & 0.8551 & 83.5984 & 706.0686 \\
2 & 0.1879 & 0.7678 & 83.6888 & 682.0075 \\
3 & 0.2234 & 0.6921 & 83.8587 & 661.1817 \\
4 & 0.2555 & 0.6196 & 84.0451 & 641.0225 \\
5 & 0.2829 & 0.5522 & 84.2541 & 621.7850 \\
6 & 0.3039 & 0.4925 & 84.4941 & 603.8023 \\
7 & 0.3110 & 0.4493 & 84.8011 & 588.5452 \\
8 & 0.3193 & 0.4128 & 85.1023 & 573.0528 \\
9 & 0.3217 & 0.3918 & 85.4321 & 558.7172 \\
10 & 0.3257 & 0.3814 & 85.7541 & 544.0679 \\
11 & 0.3196 & 0.3939 & 86.1248 & 531.3990 \\
12 & 0.3114 & 0.4202 & 86.5057 & 519.1419 \\
13 & 0.2946 & 0.4639 & 86.9281 & 508.5711 \\
14 & 0.2778 & 0.5141 & 87.3505 & 498.0003 \\
15 & 0.2563 & 0.5729 & 87.7956 & 488.3511 \\
16 & 0.2265 & 0.6411 & 88.2807 & 480.3293 \\
17 & 0.1919 & 0.7144 & 88.7890 & 473.2487 \\
18 & 0.1462 & 0.7966 & 89.3509 & 468.3446 \\
19 & 0.0908 & 0.8859 & 89.9596 & 465.3425 \\
20 & 0.0215 & 0.9849 & 90.6354 & 465.0659 \\
\hline \hline & & & & \\
\hline
\end{tabular}


Table 3 Pessimistic pareto frontier.

\begin{tabular}{lllll}
\hline \hline No. $i$ & $D_{i}^{+}$ & $L_{i}^{+}$ & $f_{\mathrm{OC}} /(\mathrm{S} / \mathrm{h})$ & $f_{\mathrm{OE}} /\left(10^{-4} \mathrm{t} / \mathrm{h}\right)$ \\
\hline 0 & -0.1493 & 1.1106 & 84.6337 & 775.8557 \\
1 & -0.0683 & 1.0031 & 84.5841 & 746.1082 \\
2 & -0.007 & 0.9110 & 84.6295 & 720.2235 \\
3 & 0.0365 & 0.8335 & 84.7608 & 697.8291 \\
4 & 0.0739 & 0.7622 & 84.9216 & 676.6307 \\
5 & 0.0962 & 0.7058 & 85.1553 & 658.3932 \\
6 & 0.1293 & 0.6433 & 85.3368 & 638.0379 \\
7 & 0.1566 & 0.5900 & 85.5464 & 618.8200 \\
8 & 0.1698 & 0.5556 & 85.8240 & 602.3668 \\
9 & 0.1798 & 0.5320 & 86.1170 & 586.5410 \\
10 & 0.1878 & 0.5193 & 86.4197 & 571.1074 \\
11 & 0.1806 & 0.5312 & 86.7957 & 558.6542 \\
12 & 0.1789 & 0.5468 & 87.1452 & 545.1226 \\
13 & 0.1668 & 0.5805 & 87.5450 & 533.6302 \\
14 & 0.1547 & 0.6206 & 87.9447 & 522.1378 \\
15 & 0.1394 & 0.6688 & 88.3598 & 511.2728 \\
16 & 0.1148 & 0.7286 & 88.8199 & 502.2315 \\
17 & 0.09 & 0.7911 & 89.2809 & 493.2293 \\
18 & 0.0516 & 0.8658 & 89.8075 & 486.8938 \\
19 & 0.0134 & 0.9414 & 90.3332 & 480.5191 \\
20 & -0.0428 & 1.0307 & 90.9458 & 477.6738 \\
\hline \hline
\end{tabular}

Table 4 Wind speed and wind farm output under 3 scenarios.

\begin{tabular}{lllllll}
\hline \hline & Scenario 1 & \multicolumn{3}{c}{ Scenario 2 } & \multicolumn{3}{c}{ Scenario 3 } \\
\cline { 2 - 7 } No & $\begin{array}{l}\text { Wind } \\
\text { speed/(m/ } \\
\text { s) }\end{array}$ & $\begin{array}{l}\text { The out } \\
\text { put of } \\
\text { wind } \\
\text { power/M } \\
\text { W }\end{array}$ & $\begin{array}{l}\text { Wind } \\
\text { speed/(m/ } / \\
\text { s) }\end{array}$ & $\begin{array}{l}\text { The out } \\
\text { put of } \\
\text { wind } \\
\text { power/M } \\
\text { W }\end{array}$ & $\begin{array}{l}\text { Wind } \\
\text { speed/(m/s) }\end{array}$ & $\begin{array}{l}\text { The out } \\
\text { put of } \\
\text { wind } \\
\text { power/M } \\
\text { W }\end{array}$ \\
\hline 1 & 7.5537 & 24.23 & 2.9577 & 0 & 6.8269 & 19.27 \\
2 & 18.5753 & 150 & 13.3952 & 118.74 & 14.1257 & 127.51 \\
3 & 16.6388 & 200 & 11.5184 & 139.45 & 17.3843 & 200 \\
4 & 9.4143 & 36.92 & 9.9466 & 40.54 & 10.0125 & 40.99 \\
5 & 12.2464 & 149.93 & 9.0771 & 92.31 & 16.7968 & 200 \\
6 & 6.7844 & 51.61 & 3.9627 & 13.13 & 3.5529 & 7.54 \\
7 & 12.4836 & 172.43 & 12.4084 & 171.06 & 11.9494 & 162.72 \\
8 & 10.4075 & 43.69 & 8.0027 & 27.29 & 7.8734 & 26.41 \\
\hline \hline
\end{tabular}

frontier in the actual wind speed scenario will also be distributed between the optimistic and pessimistic Pareto frontier. That is, the optimistic and pessimistic Pareto frontier contains all the possibilities of the actual Pareto frontier.

Obviously, compared with the existing stochastic economic scheduling and robust economic scheduling, the model and method proposed in this paper can effectively deal with multiple objective functions of economic scheduling and achieve the comprehensive coordination and optimization of economy and environment. Compared with the traditional multi-objective economic scheduling, the wind speed is modeled as an interval variable, which can

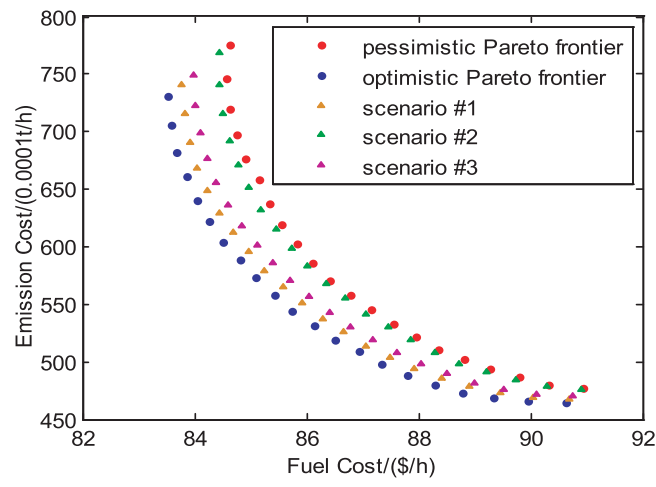

Fig. 4 The relationship among optimistic, pessimistic and actual Pareto frontiers.

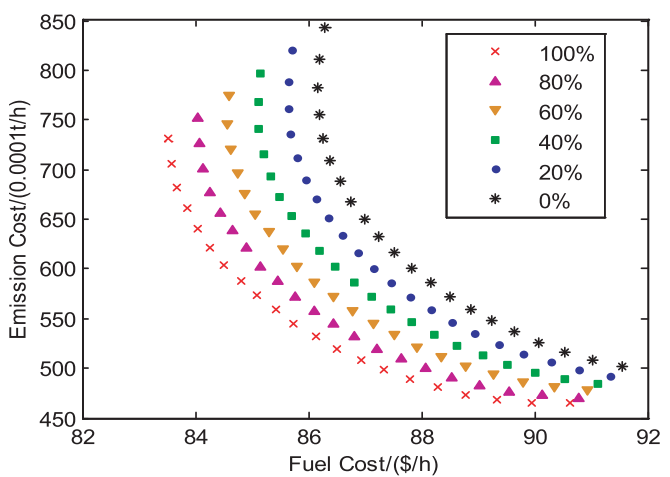

Fig.5 The capacity of wind power effect on optimistic Pareto frontier.

consider the influence of wind power uncertainty on economic scheduling results. As shown in Fig. 4, in the traditional multi-objective economic dispatching method, the wind power is taken as the deterministic quantity in scenerio \#1, \#2, \#3, and the Pareto frontier obtained ignores the uncertainty of wind power, therefore, the practical applicability of the economic dispatching results obtained are questionable.

\subsection{Impact of Wind Power Access Capacity on the Results}

In Fig. 5 and Fig. 6 are shown the optimistic Pareto frontier and the pessimistic Pareto frontier of each wind farm in Table 1 with proportional access to the power system. In Table 5 is a compromise solution for the multi-objective interval power generation scheduling under the different wind power access levels. As the proportion of wind power increases from $0 \%$ to $100 \%$, the optimistic Pareto frontier and the pessimistic Pareto frontier gradually close to the axis, indicating that the wind power access reduces the economic cost and environmental cost of the thermal power plant.

As the proportion of wind power access decreases from $100 \%$ to $0 \%$, the interval uncertainty of the power system gradually decreases, and the distance between the optimistic Pareto frontier and the pessimistic Pareto frontier gradually shrinks, eventually narrowing to a Pareto frontier. When the proportion of wind power is $0 \%$, there is no un- 


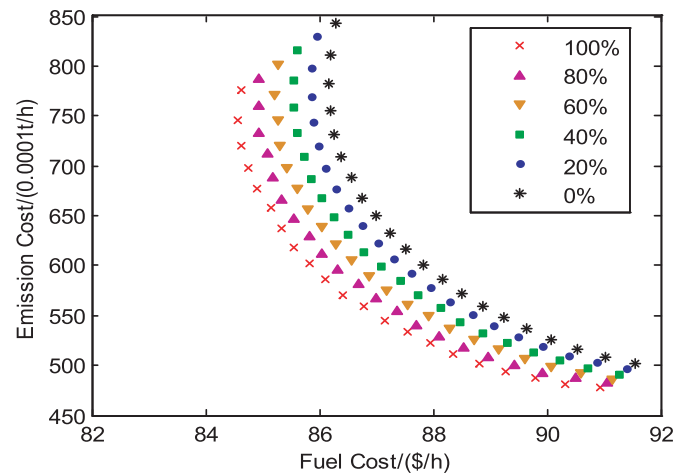

Fig. 6 The capacity of wind power effect on pessimistic Pareto frontier.

Table 5 Compromise solution under different wind power level.

\begin{tabular}{ccccc}
\hline \hline $\begin{array}{c}\text { Wind } \\
\text { Capacitanc } \\
\mathrm{e} / \%\end{array}$ & $f_{O C} /(\$ / \mathrm{h})$ & $f_{O E} /(10-4 \mathrm{t} / \mathrm{h})$ & $\mathrm{fPC} /(\$ / \mathrm{h})$ & $f_{P E} /\left(10^{-4} / \mathrm{h}\right)$ \\
\hline 100 & 85.7541 & 544.0679 & 86.4197 & 571.1074 \\
80 & 86.0944 & 557.8916 & 86.9932 & 566.6739 \\
60 & 86.4400 & 571.9309 & 86.8695 & 589.3821 \\
40 & 86.8015 & 586.6173 & 87.0959 & 598.5782 \\
20 & 87.4763 & 586.3015 & 87.6226 & 592.2427 \\
0 & 87.8325 & 600.7722 & 87.8325 & 600.7722 \\
\hline \hline
\end{tabular}

certainty in the power system without the wind power gridconnection considered. The multi-target interval power generation scheduling degenerates into a deterministic multiobjective power generation scheduling problem, and the curves of the optimistic Pareto frontier and the pessimistic Pareto frontier will overlap together. Accordingly, the optimistic Pareto compromise solution and pessimistic Pareto compromise solution obtained by the distance assessment methods will also coincide at a point, as shown in Table 5, and $f_{P C}=f_{O C}=87.8325(\$ / \mathrm{h}), f_{P E}=f_{O E}=600.7722$ $\left(10^{-4} \mathrm{t} / \mathrm{h}\right)$.

\section{Conclusion}

In this paper, a study of the dispatching problems of windthermal power system is made, and a multi-objective interval generation dispatching model considering wind power is established, and an improved normal boundary intersection method is utilized to solve the model. The calculation results of the actual system of 16-unit 174-bus show that:

(1) An uniform optimistic and pessimistic Pareto optimal solution set can be obtained by the established model and proposed method, which can be used to quantitatively analyze the impact of wind power on the economic and environmental cost of power generation dispatching.

(2) The actual Pareto frontier in the actual wind speed scene is distributed between the optimistic and pessimistic Pareto frontiers, which contains all the possibilities of the actual Pareto frontier.

(3) The distance between the optimistic and pessimistic Pareto frontiers can well reflect the uncertainty of the system interval, and the larger the scale of wind power grid connected, the stronger the interval uncertainty of power system, and the greater the distance between the optimistic and pessimistic Pareto frontiers.

In addition, the proposed improved normal boundary intersection method has good portability and robustness. We will use it to solve multi-objective interval unit commitment and other problems in the future work.

\section{Acknowledgments}

This work was supported by National Natural Science Foundation of China (61561007) and the Natural Science Foundation of Guangxi in China (2018GXNSFAA294019).

\section{References}

[1] Y. Mao and W. Shaoshuai, "A Review of Wind Power Forecasting \& Prediction," International Conference on Probabilistic Methods Applied to Power Systems (PMAPS), IEEE, pp.297-303, 2016.

[2] N. Amjady, F. Keynia, and H. Zareipour, "Wind Power Prediction by a New Forecast Engine Composed of Modified Hybrid Neural Network and Enhanced Particle Swarm Optimization," IEEE Trans. Sustain. Energy, vol.2, no.3, pp.265-276, July 2011.

[3] A. Martinez-Mares and C.R. Fuerte-Esquivel, "A Robust Optimization Approach for the Interdependency Analysis of Integrated Energy Systems Considering Wind Power Uncertainty," IEEE Trans. Power Syst., vol.28, no.4, pp.3964-3976, Nov. 2013.

[4] Y.F. Cao and X. Cai, "Optimal energy storage system allocation and operation for improving wind power penetration," IET Generation, Transmission \& Distribution, vol.9, no.16, pp.2672-2678, Nov. 2015.

[5] X. Ran, S. Miao, Z. Jiang, and H. Xu, "A framework for uncertainty quantification and economic dispatch model with wind-solar energy," International Journal of Electrical Power \& Energy Systems, vol.73, pp.23-33, 2015.

[6] J. Hetzer, D.C. Yu, and K. Bhattarai, "An Economic Dispatch Model Incorporating Wind Power," IEEE Trans. Energy Convers., vol.23, no.2, pp.603-611, June 2008.

[7] A.H. Shahirinia, E.S. Soofi, and D.C. Yu, "Probability distributions of outputs of stochastic economic dispatch," International Journal of Electrical Power \& Energy Systems, vol.81, pp.308-316, Oct. 2016.

[8] X. Liu, "Emission minimisation dispatch constrained by cost and wind power," IET Generation, Transmission \& Distribution, vol.5, no.7, pp.735-742, July 2011.

[9] D. Bienstock, M. Chertkov, and S. Harnett, "Chance Constrained Optimal Power Flow: Risk-Aware Network Control under Uncertainty," Siam Review, vol.56, no.3, pp.461-495, Sept. 2012.

[10] X. Liu and W. Xu, "Economic load dispatch constrained by wind power availability: a here-and-now approach," IEEE Trans. Sustain. Energy, vol.1, no.1, pp.2-9, May 2010.

[11] X. Liu and W. Xu, "Economic Load Dispatch Constrained by Wind Power Availability: A Wait-and-See Approach," IEEE Trans. Smart Grid, vol.1, no.3, pp.347-355, Dec. 2010.

[12] V. Miranda and P.S. Hang, "Economic dispatch model with fuzzy wind constraints and attitudes of dispatchers," IEEE Trans. Power Syst., vol.20, no.4, pp.2143-2145, Nov. 2005.

[13] T. Ding, R. Bo, F. Li, and H. Sun, "A Bi-Level Branch and Bound Method for Economic Dispatch With Disjoint Prohibited Zones Considering Network Losses," IEEE Trans. Power Syst., vol.30, no.6, pp.2841-2855, Nov. 2015.

[14] Y. Wang, Q. Xia, and C. Kang, "Unit Commitment With Volatile Node Injections by Using Interval Optimization," IEEE Trans. Power Syst., vol.26, no.3, pp.1705-1713, Aug. 2011. 
[15] H. Bao, W. Hua, and X. Guo, "Interval optimal power flow calculation for AC/DC power system considering renewable energy source," Proc. CSEE, vol.35, no.16, pp.4006-4014, Aug. 2015.

[16] M. Zhang, Z. Hu, and M. Hu, "Robust Economic Dispatch Considerring Wind Uncertainty," International Conference on Renewable Power Generation (RPG), IET, pp.1-5, 2015.

[17] D. Bertsimas, E. Litvinov, X.A. Sun, J. Zhao, and T. Zheng, "Adaptive Robust Optimization for the Security Constrained Unit Commitment Problem," IEEE Trans. Power Syst., vol.28, no.1, pp.52-63, 2013.

[18] R. Jiang, J. Wang, and Y. Guan, "Robust Unit Commitment with Wind Power and Pumped Storage Hydro," IEEE Trans. Power Syst., vol.27, no.2, pp.800-810, 2012.

[19] I. Ziane, F. Benhamida, A. Graa, and Y. Salhi, "Wind Power Impact in the Environmental/Economic Dispatch," 2015 4th International Conference on Electrical Engineering (ICEE), IEEE, pp.13-15, 2015.

[20] M.-R. Andervazh and S. Javadi, "Emission-economic dispatch of thermal power generation units in the presence of hybrid electric vehicles and correlated wind power plants," IET Generation, Transmission \& Distribution, vol.11, no.9, pp.2232-2243, Feb. 2017.

[21] W. Wei, J. Wang, and S. Mei, "Convexification of the Nash Bargaining Based Environmental-Economic Dispatch," IEEE Trans. Power Syst., vol.31, no.6, pp.5208-5209, Jan. 2016.

[22] B. Wang, S. Wang, X.-Z. Zhou, and J. Watada, "Two-Stage MultiObjective Unit Commitment Optimization Under Hybrid Uncertainties," IEEE Trans. Power Syst., vol.31, no.3, pp.2266-2277, May 2016.

[23] R. Kun and J. Qu, "A Multiobjective Interval Programming Model for Wind-Hydrothermal Power System Dispatching Using 2-Step Optimization Algorithm," Scientific World Journal, pp.1-9, 2014.
[24] Z. Zhou, B. Wang, B. Gu, B. Ai, S. Mumtaz, J. Rodriguez, and M. Guizani, "Time-dependent pricing for bandwidth slicing under information asymmetry and price discrimination," IEEE Trans. Commun., pp.1-17, 2020.

[25] B. Gu and Z. Zhou, "Task offloading in vehicular mobile edge computing: A matching-theoretic framework," IEEE Veh. Technol. Mag., vol.14, no.3, pp.100-106, 2019.

[26] I. Das and J.E. Dennis, "Normal-boundary intersection: a new method for generating the Pareto surface in nonlinear multicriteria optimization problems," SIAM Journal on Optimization, vol.8, no.3, pp.631-657, March 1998.

[27] C. Roman and W. Rosehart, "Evenly distributed pareto points in multi-objective optimal power flow," IEEE Trans. Power Syst., vol.21, no.2, pp.1011-1012, June 2006.

[28] Y. Fu, M. Liu, and L. Li, "Multiobjective Stochastic Economic Dispatch with Variable Wind Generation Using Scenario-Based Decomposition and Asynchronous Block Iteration," IEEE Trans. Sustain. Energy, vol.7, no.1, pp.139-149, Oct. 2016.

[29] B. Colson, P. Marcotte, and G. Savard, "Bilevel programming: A survey," A Quarterly Journal of Operations Research, vol.3, no.2, pp.87-107, June 2005.

[30] F. Capitanescu, M. Glavic, D. Ernst, and L. Wehenkel, "Interior-point based algorithms for the solution of optimal power flow problems," Electric Power Systems Research, vol.77, no.5-6, pp.508-517, April 2007.

[31] S.J. Kazempour, A.J. Conejo, and C. Ruiz, "Strategic Generation Investment Using a Complementarity Approach," IEEE Trans. Power Syst., vol.26, no.2, pp.940-948, May 2011.

[32] M.A. Abido, "A niched Pareto genetic algorithm for multiobjective environmental/economic dispatch," International Journal of Electrical Power \& Energy Systems, vol.25, no.2, pp.97-105, Feb. 2003.

\section{Appendix: Data of the Study Cases}

Table A. 1 Economic cost parameters and emission gas cost parameters of thermal power unit.

\begin{tabular}{|c|c|c|c|c|c|c|c|c|c|c|}
\hline $\mathrm{N}$ & $\underline{P}_{T i} / \mathrm{MW}$ & $\bar{P}_{T i} / \mathrm{MW}$ & $\mathrm{a} /\left(\$ / \mathrm{MW}^{2} \mathrm{~h}\right)$ & $\mathrm{b} /(\$ / \mathrm{MWh})$ & $\mathrm{c} /(\$ / \mathrm{h})$ & $\alpha /\left(10^{-4} \mathrm{t} / \mathrm{MW}^{2} \mathrm{~h}\right)$ & $\beta /\left(10^{-4} \mathrm{t} / \mathrm{MWh}\right)$ & $\gamma /\left(10^{-4} \mathrm{t} / \mathrm{h}\right)$ & $\delta /(\mathrm{t} / \mathrm{h})$ & $\lambda / \mathrm{MW}-1$ \\
\hline 1 & 100 & 600 & 0.10 & 1.25 & 0.01 & 4.091 & -5.217 & 6.490 & $2.0 \times 10-6$ & 0.02857 \\
\hline 2 & 60 & 200 & 0.12 & 2.6 & 0.05 & 2.543 & -6.022 & 5.638 & $5.0 \times 10-6$ & 0.03333 \\
\hline 3 & 50 & 300 & 0.10 & 1.5 & 0.05 & 4.258 & -5.094 & 4.586 & $1.0 \times 10-8$ & 0.08000 \\
\hline 4 & 100 & 400 & 0.10 & 1.5 & 0.10 & 5.326 & -3.557 & 3.380 & $2.0 \times 10-5$ & 0.02000 \\
\hline 5 & 100 & 400 & 0.10 & 2.1 & 0.10 & 4.313 & -5.192 & 4.511 & $1.0 \times 10-8$ & 0.08000 \\
\hline 6 & 60 & 300 & 0.14 & 2.0 & 0.20 & 5.171 & -5.555 & 5.151 & $1.0 \times 10-7$ & 0.06667 \\
\hline 7 & 50 & 300 & 0.10 & 1.6 & 0.10 & 4.132 & -5.554 & 5.992 & $2.0 \times 10-6$ & 0.02857 \\
\hline 8 & 50 & 400 & 0.10 & 1.5 & 0.05 & 3.523 & -6.047 & 4.831 & $5.0 \times 10-6$ & 0.03333 \\
\hline 9 & 100 & 500 & 0.10 & 1.5 & 0.01 & 2.256 & -5.094 & 4.880 & $1.0 \times 10-8$ & 0.08000 \\
\hline 10 & 100 & 500 & 0.10 & 1.5 & 0.01 & 5.326 & -3.551 & 2.985 & $2.0 \times 10-5$ & 0.02000 \\
\hline 11 & 100 & 800 & 0.10 & 1.0 & 0.01 & 2.248 & -5.110 & 4.595 & $1.0 \times 10-8$ & 0.08000 \\
\hline 12 & 100 & 600 & 0.10 & 1.23 & 0.20 & 6.958 & -5.554 & 5.152 & $1.0 \times 10-7$ & 0.06667 \\
\hline 13 & 100 & 800 & 0.10 & 1.2 & 0.10 & 4.091 & -5.554 & 6.514 & $2.0 \times 10-6$ & 0.02857 \\
\hline 14 & 100 & 400 & 0.10 & 1.6 & 0.05 & 2.576 & -6.021 & 5.638 & $5.0 \times 10-6$ & 0.03333 \\
\hline 15 & 40 & 200 & 0.12 & 2.5 & 0.05 & 4.119 & -5.094 & 4.021 & $1.0 \times 10-8$ & 0.08000 \\
\hline 16 & 40 & 200 & 0.11 & 2.4 & 0.10 & 5.736 & -3.551 & 3.380 & $2.0 \times 10-5$ & 0.02000 \\
\hline
\end{tabular}


Table A. 2 Parameter of wind farms.

\begin{tabular}{|c|c|c|c|c|c|c|}
\hline 1 & 20 & 0.75 & 4.0 & 25 & 15.0 & {$[2.4,7.7]$} \\
\hline 3 & 10 & 2.00 & 3.5 & 30 & 15.0 & {$[11.5,18.7]$} \\
\hline 4 & 15 & 0.75 & 4.0 & 25 & 15.0 & {$[9.4,12.0]$} \\
\hline 5 & 10 & 2.00 & 4.0 & 30 & 15.0 & {$[5.5,17.6]$} \\
\hline 8 & 25 & 0.75 & 4.0 & 25 & 15.0 & {$[6.8,11.3]$} \\
\hline
\end{tabular}

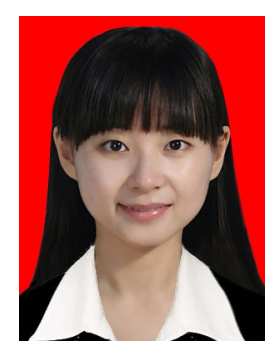

Xiaoxuan Guo was born in Guilin of Guangxi Province, China, in 1986. She received her B.Eng. from the School of Automation and Electrical Engineering, University of Jinan, China. She received her M.Eng and $\mathrm{Ph}$.D. degree from the School of Electrical Engineering, Guangxi University, China. Her main research interests include optimal operation of power system, and the power system analysis.

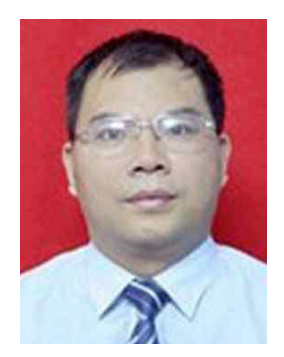

Renxi Gong was born in Guilin of Guangxi Province, China in 1962. From August 1990 to March 1993, he was a graduate student majoring in Semiconductor Devices and Microelectronics at Xidian University. He received his M.S. degree in March 1993. From 1994 to 1997, he worked as an assistant at Guangxi University, and from February 1998 to January 2002, he worked on his Ph.D degree in Microelectronics and Solid-state Electronics at Xidian University. He is currently a professor at Electrical Engineering School of Guangxi University. His present research interests are intelligent detection technology, power electronic technology, renewable source generation technology and their applications. (corresponding author: rxgong@gxu.edu.cn)

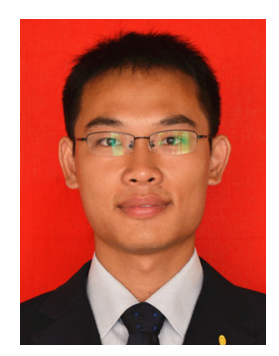

Haibo Bao was born in Hefei of Anhui Province, China, in 1988. He received his B.Eng. from the School of Electronic Science and Technology, Anhui University, China. He received his M.Eng and Ph.D. degree from the School of Electrical Engineering, Guangxi University, China. Now he works at China Southern Power Grid Company. His research interests are optimal operation of power system, and the power system analysis.

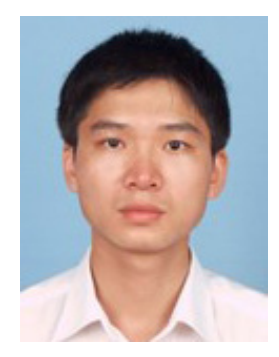

Zhenkun Lu received the B.S. degrees from Wuhan University, Wuhan, China, in 2003, the M.S. degrees from Guangxi Normal University, Guilin, China, in 2009, Ph.D. degrees from South China University of Technology (SCUT), Guangzhou, China, in 2013. He was a visiting scholar at the University of Arizona, Tucson, USA, from March 2016 to March 2017. He is now a professor with Guangxi University for Nationalities, Nanning, Guangxi, China. His research interests are digital signal processing

and image processing. 